\title{
On the Intrinsic Simplicity of Spectral Variability of GRBs
}

\author{
A. Chernenko
}

\begin{abstract}
In this paper we present a Multi-Scale Correlation Analysis (MSCA) of the light curves of gamma-ray bursts recorded in different energy ranges. This analysis allows us to identify time intervals where emission variability can be reduced to a single physical parameter and can therefore be robustly attributed to a single physical emitter. The properties of these intervals can then be investigated separately, and the spectral properties of individual emitters can be analysed. The signatures of hidden dynamical relations between individual emitters are also discussed.
\end{abstract}

Keywords: Gamma-ray bursts, variability, multi-scale, correlations, random matrices, eigenvalues.

\section{Introduction: dimension of spectral variability}

Relativistically expanding sources produce radiation that manifests a rich spectral evolution and diverse light curve structures. Even if individual emitting regions have a similar and simple geometry and rest frame spectra, spectral and temporal evolution within any period of an observer's time will be very diverse, since many individual emitters contribute to this period at different viewing angles and Lorentz factors, at different stages of development. In reality, the physical properties and even the physical nature of individual emitters within a single source can also be quite diverse.

In this situation we believe that the very first stage of spectroscopy should be model independent, and should give a clear understanding of the following:

1. The dimension of variability for the entire period of the observations - how many spectral parameters are in fact independently variable during the period of the observations.

2. Segmentation into low-dimension periods - can the entire period of the observations be split into sub-periods for each of which the internally measured dimension of variability is considerably lower than the dimension for the entire period

3. Hidden dynamics - if there is evidence that the emission properties at any moment of the observations are dependent on its properties at earlier moment(s) of time. If such signatures are found, can the dimension of variability be further decreased by taking into account the hidden dynamics.

In this paper I will focus on the first two items on this list.

\section{Measuring the dimension of variability by correlating light curves}

Correlation analysis of emission light curves recorded in different energy ranges (wave lengths) is a natural, and model-independent way of estimating the dimension of spectral variability. The rank $R_{C M}$ of the corresponding correlation matrix $\mathbf{C M}$ is exactly its numerical measure. Figure (1) presents the light curves of GRB911118. These light curves measured in $p=8$ energy ranges by BATSE/COMPTON Large Area Detectors with time resolution of $0.016 \mathrm{~s}$ produced a data matrix with dimension $p \times n=8 \times 1000$.

Figure (2) presents the corresponding correlation matrix. The spectrum of its sorted eigenvalues $E V(i)$ $(i=1, \ldots, 8)$ is also presented. It is clear that there are 2 non-zero eigenvalues, and therefore the rank of the correlation matrix $R_{C M}=2$. In this case, we would say that spectral variability is twodimensional.

This original result was first obtained by Chernenko and Mitrofanov in [1] and was interpreted as evidence for two non-correlated emission components related to physically distinct emission sources. Later similar results were obtained in a number of papers, e.g. [2]. There is also an alternative interpretation involving a single emission component with two independently variable parameters [3].

The next logical step in the investigation, as was noted in the introduction, is segmentation, i.e. to look at the GRB through time windows of different widths and positions in order to find sub-intervals where the spectral variability is just one-dimensional, i.e. the covariance matrix has just a single non-zero eigenvalue. We call this approach Multi-Scale Correlation Analysis, and will present its results later 


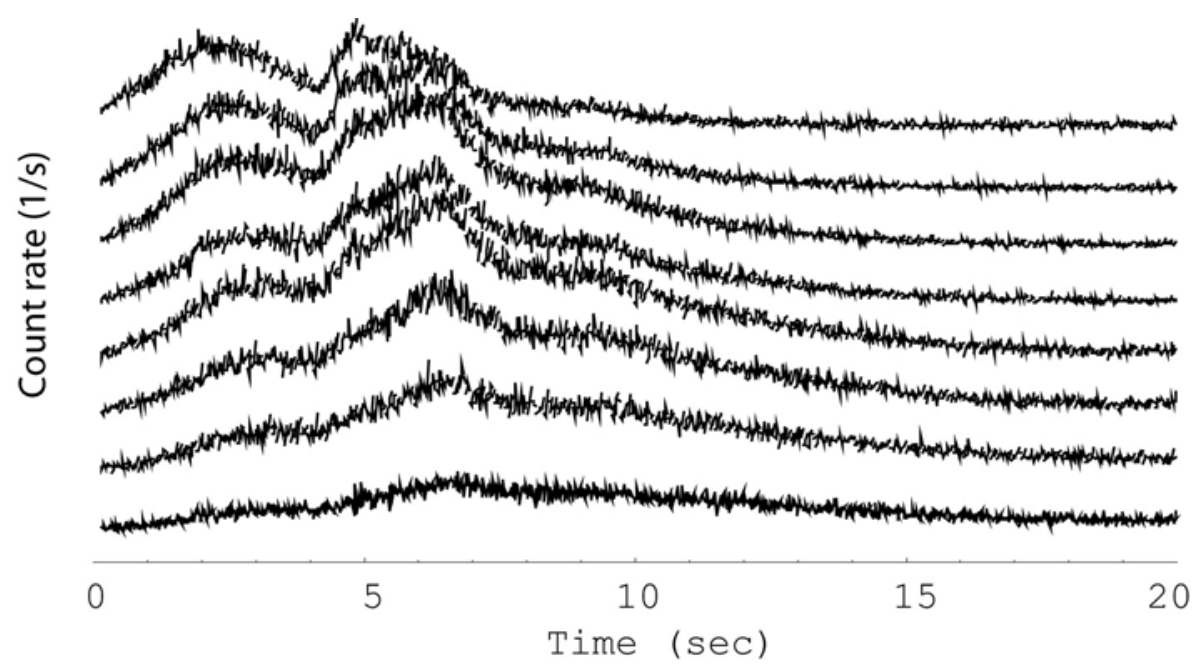

Fig. 1: Gamma-ray light curves of gamma-ray burst GB911118 detected by the BATSE/COMPTON experiment are presented as measured in 8 broad energy channels between $37 \mathrm{keV}$ and approx. $2 \mathrm{MeV}$ with time resolution of 0.016 seconds. The amplitudes of the light curves are arbitrarily scaled to emphasize their shapes
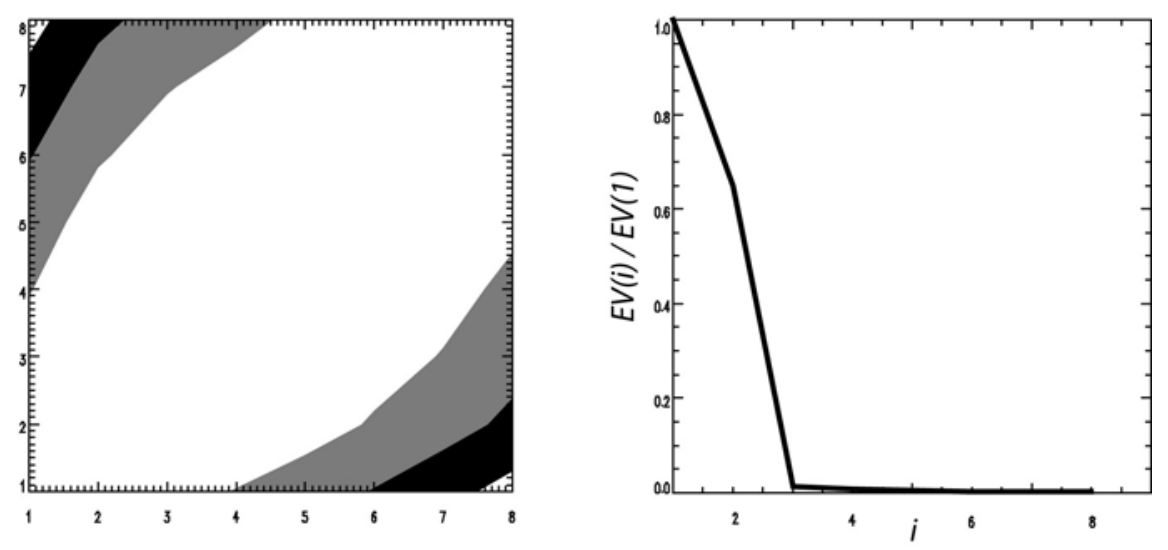

Fig. 2: Correlation matrix calculated for the 8 light curves of GB011118 presented in Figure (1) is graphically shown in the left panel. In the right panel, the spectrum of its sorted eigenvalues $E V(i)$ is presented. The eigenvalues are normalized by the largest $E V(1)$. Only the two largest eigenvalues are evidently non-zero, so the rank of the correlation matrix $R_{C M}=2$

in this paper. But before that two important statements should be put forward:

- As our goal is to study sub-intervals of the GRB time history on multiple time scales down to the shortest ones where covariance can be calculated (i.e. $n \rightarrow 3$ ), we need to deal with cases when $n<p$ and the covariance matrix is $p-n+1$ times singular.

- In the real world, times series are "contaminated" by Poisson noise. Therefore, to determine the number of eigenvalues which are significantly larger than zero we need to know how to propagate Poisson noise from raw time series to noise in eigenvalues.

\section{Statistical properties of eigenvalues of correlation matrices for Poisson noise time series of arbitrary duration}

Let us consider a data matrix with dimensions $n \times p$, where $p$ is number of time series and $n$ is number of measurements (duration) for each of the time series. Let us assume that each of the time series is a stationary Poisson process with parameter $\lambda$. Then, without loss of generalization we can transform the Poisson process by means of the Anscombe formula [4] to 
a nearly Gaussian process with standard deviation $\sigma=1$ :

$$
A: x \Rightarrow 2 \sqrt{x+\frac{3}{8}}
$$

The correlation matrices for Gaussian time series with $\sigma=1$ are known in random matrices theory as Wishart matrices. Analytical results on their eigenvalues are basically limited to the quasi-continuous density distribution of the eigenvalues in the asymptotic case when $p \leq n \rightarrow \infty$, which is given by the Marcenko-Pastur density function [5]. Analytical results on the distribution of individual eigenvalues are basically limited to the density distribution of the largest eigenvalue, which is described by the TracyWisdom law [7]. Although this law was found to be reasonably accurate for $n$ and $p$ as small as 5 [6], for our investigation this is not sufficient since $i$ ) we need to know the statistical properties of smaller eigenvalues in order to determine the dimension of spectral variability and ii) the Tracy-Wisdom law holds for $n \geq p$, which poses an unnecessary restriction on our analysis.

To sum up, in the case of $n \ll \infty$ and especially when $n \lesssim p \ll \infty$, when the correlation matrix becomes singular, existing analytical results are insufficient. Therefore, we attempted a number of Monte-Carlo simulations to derive the sample distribution for eigenvalues for data matrices with different $n:[4,8, \ldots, 1024]$ and $p:[4,8,12,16,32,64]$.
For each realization of data matrix $D$ we computed, by means of SVD, the spectrum of eigenvalues. For each pair of $n$ and $p$, the number of such random realizations was $10^{7}$. In Figure (3) we present the probability distributions for non-singular eigenvalues for some values of $n$ and $p$.

The goal of this Monte-Carlo experiment was to estimate the one-sided confidence limits $E V_{i}^{p}$ for sorted eigenvalues $E V_{i}, i:[1, n]$ for a typical set of $\alpha$ values: $\alpha$ : $\left[0.1,10^{-2}, 10^{-3}, 10^{-4}, 10^{-5}\right]$. In Figure (4) these confidence limits are presented as functions of $n$.

\section{The quest for one-dimensional spectral variability}

Again, mathematically speaking, one-dimensional spectral variability during some time interval $\left[t_{1}, t_{2}\right]$ takes place if a correlation matrix built from multichannel time histories over this time interval has only one non-zero eigenvalue. In a real case when the Poisson background is present, there will be no zero eigenvalues. Replacing the "one non-zero eigenvalue" by "one significant eigenvalue" would not be correct because if the $\mathrm{S} / \mathrm{N}$ ratio is low, then even if the first few eigenvalues are quite comparable, only the first one could eventually be significant.
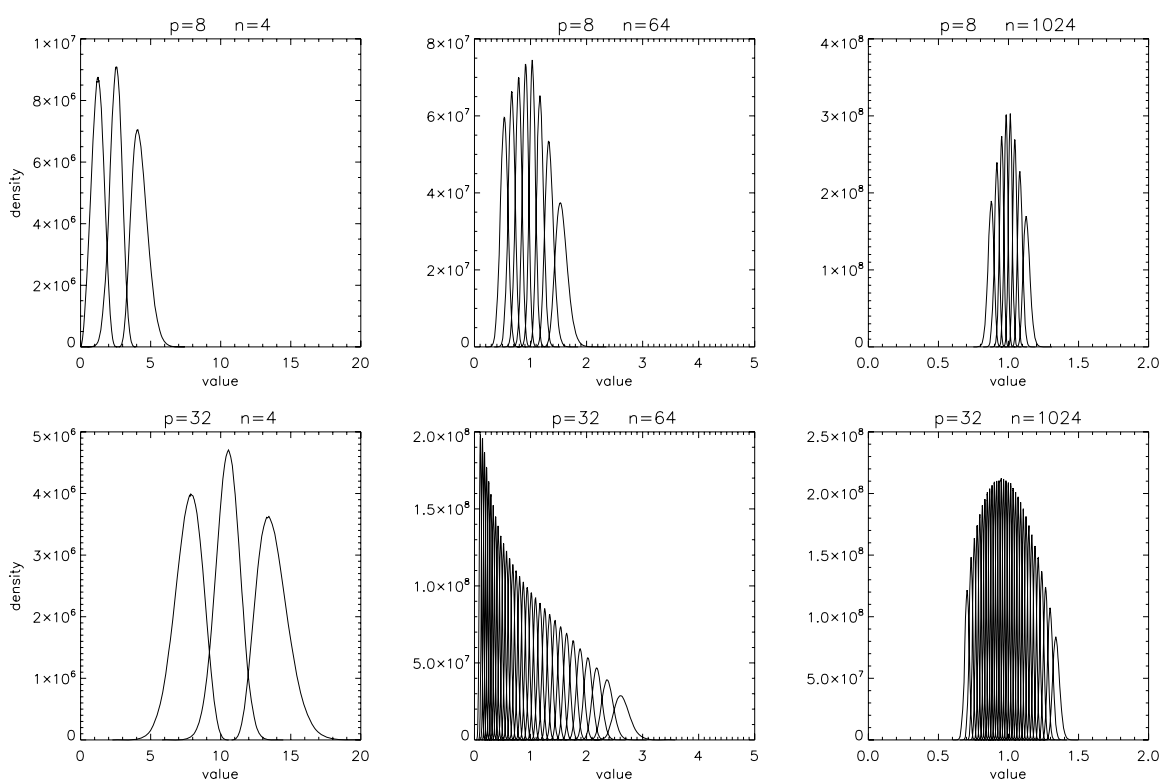

Fig. 3: Probability distributions for individual sorted non-singular eigenvalues of sample correlation matrices for Poisson noise time series are presented for some values of time series number $p$ and lengths $n$. The leftmost panels correspond to cases $n<p$ when the correlation matrices are $p-n+1$ times singular and only $n-1$ largest eigenvalues are non-zero. The rightmost panels correspond to asymptotic cases when $P \ll n \rightarrow \infty$. In this case, the eigenvalues are densely and symmetrically grouped around one, as expected from the Marcenko-Pastur density function [5] 

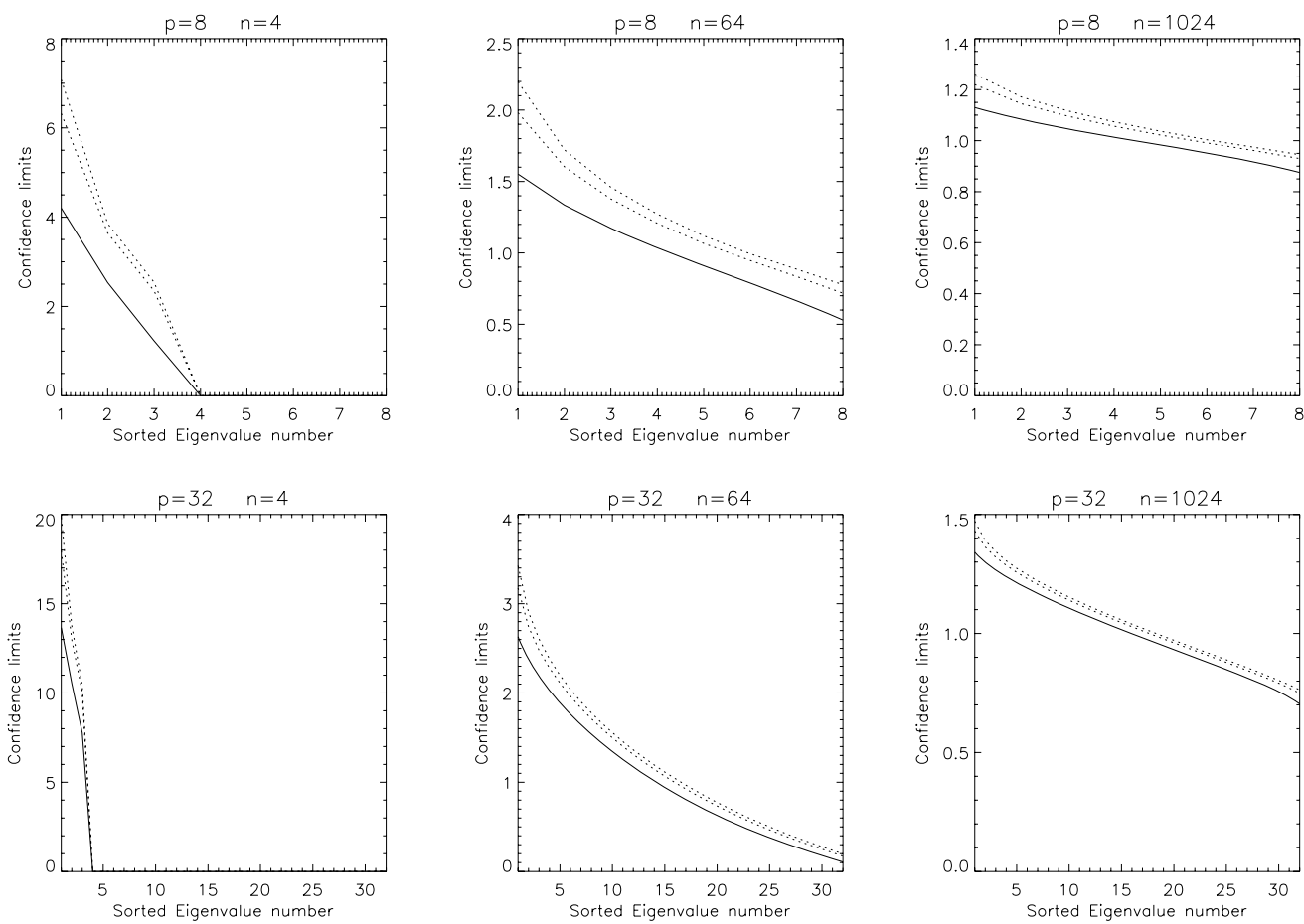

Fig. 4: Upper confidence limits are presented for the individual sorted non-singular eigenvalues of sample correlation matrices as derived from the corresponding probability distribution presented in Figure (3). The solid lines correspond to $p=0.5$ representing therefore the most probable values of the eigenvalues, while the two dotted lines in each plot correspond to $p=10^{-3}$ and $p=10^{-5}$

Thus, for the purposes of this analysis the following definition of one-dimensionality will be used:

1. the first two eigenvalues are significant with a predefined significance level of $\alpha$ and

2. their ratio $E V(1 / 2)=E V(1) / E V(2)$ is greater than a predefined level of $\epsilon$.

None of the BATSE Gamma-ray bursts that we have studied manifested one-dimensional spectral variability during its entire duration [3]. Therefore, following the idea proposed in the Introduction, we attempted segmentation - an analysis of the subperiods to find out whether one-dimensional variability exists on shorter times scales somewhere during a gamma-ray burst.

\subsection{Segmentation: looking for intervals of one-dimensional spectral variability}

Gamma-ray bursts may last from a fraction of a second to hundreds of seconds, manifesting complicated temporal structures on different time scales. To identify periods of one-dimensional spectral variability in such a complex phenomenon we have, in principle, to use a truly redundant multi-scale approach. In the case of multi-scale correlation analysis this means that the correlation matrices should be sampled for all possible time windows $\left[t_{i}, t_{j}\right]$, where $i, j:[1 . . N]$,
$N$ being the total number of time intervals for a given GRB.

More precisely, the procedure of Multi-Scale Correlation Analysis was as follows:

- For a moment $\mathrm{t}$ and time scale $\tau$, the correlation matrix $C M(t, \tau)$ size $n \times n$ is calculated for light curves in $n$ energy channels for the time interval $[t-\tau / 2, t+\tau / 2]$

- First two largest eigenvalues $E V(1, t, \tau)$ and $E V(2, t, \tau)$ of $C M(t, \tau)$ are calculated

- If both $E V(1, t, \tau)$ and $E V(2, t, \tau)$ are significant then the ratio $E V(1 / 2, t, \tau)=E V(1) / E V(2)$ is calculated as a local measure of the variability dimension at moment $t$. If $E V(1 / 2) \gg 1$ we conclude that the dimension of variability at moment $t$ and time scale $\tau$ is equal to 1 .

For the time scale of $\tau=1 \mathrm{~s}$ the result of this procedure is illustrated in Figure (5).

This procedure is then performed for a number of time scales, and for each moment $t$ the scale $\tau_{\max }$, which maximizes $E V(1 / 2, t, \tau)$ is determined:

$$
E V(1 / 2, t)_{\max }=\max _{\tau}(E V(1 / 2, t, \tau))
$$

Peaks of $E V(1 / 2, t)_{\max }$ mark time intervals $[t-$ $\left.\tau_{\max } / 2, t+\tau_{\max } / 2\right]$ where one-dimensional variability is most prominent. In Figure (6) the time histories of $E V(1 / 2, t)_{\max }$ and $\tau_{\max }(t)$ are presented together with the original multi-channel light curves 


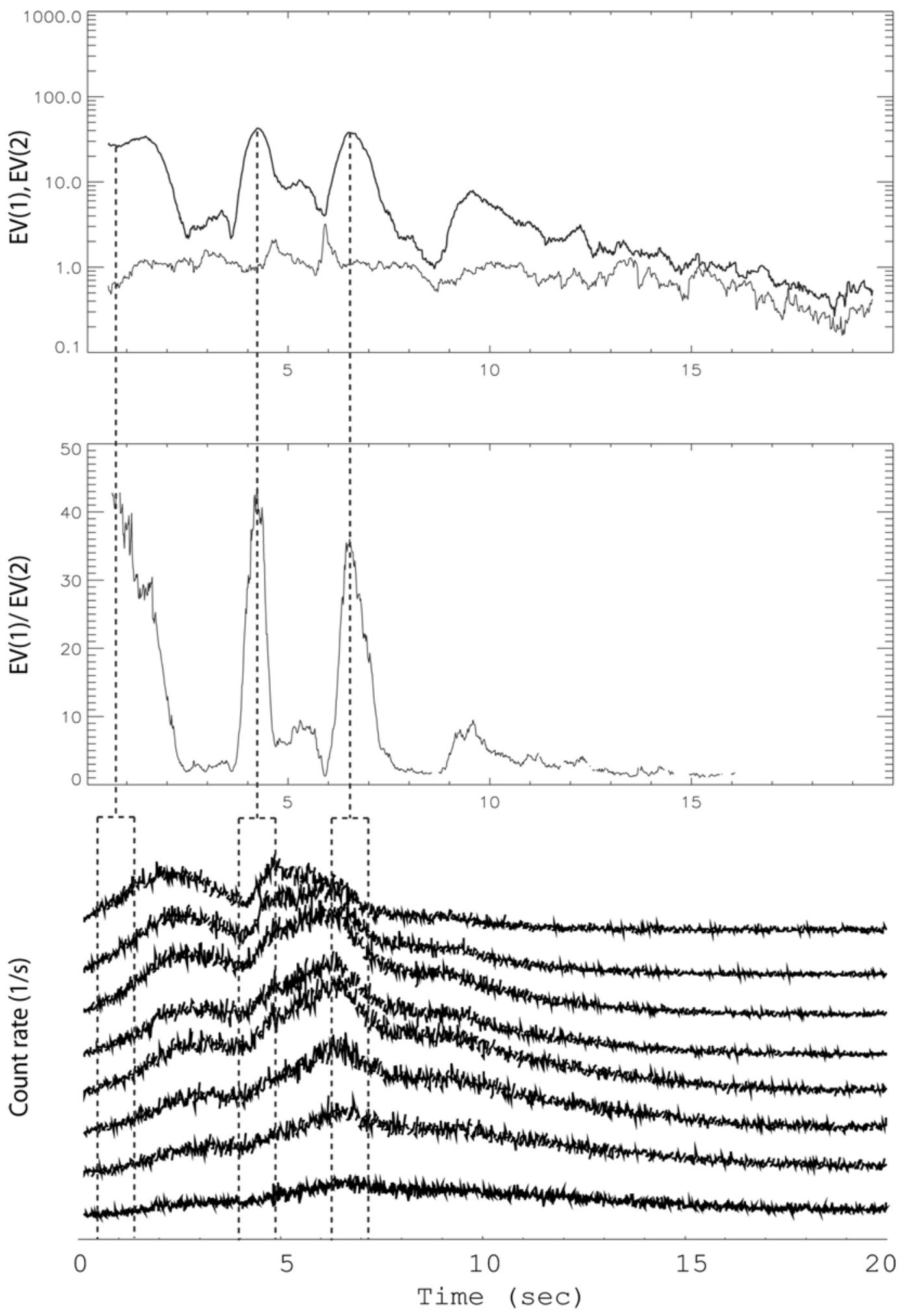

Fig. 5: Segmentation of the GB911118 light curves into sub-intervals with one-dimensional variability is demonstrated using Multi-Scale Correlation Analysis on the single time scale $\tau=1 \mathrm{~s}$. The upper panel presents the time histories of the two largest eigenvalues $E V(1, t)$ and $E V(2, t)$. The ratio $E V(1 / 2, t)$ for the same time scale $\tau=1 \mathrm{~s}$ is presented in the middle panel. The peaks of this ratio mark the center points of sub-intervals with one-dimensional variability. The bottom panel shows three most pronounced sub-intervals with one-dimensional variability in the original multi-channel light curves of GB911118 by means of dashed lines 


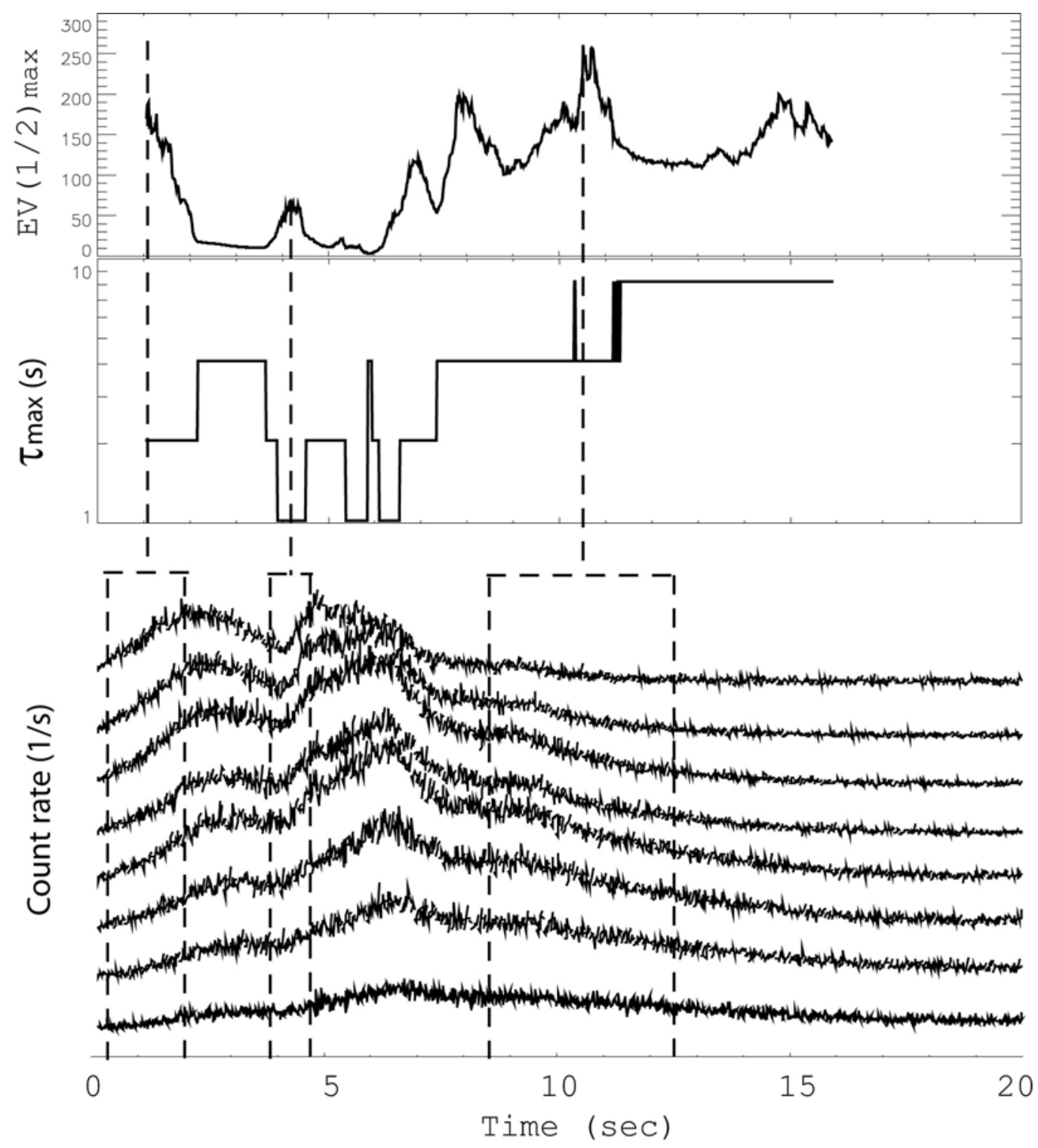

Fig. 6: Segmentation of the GB911118 light curves into sub-intervals with one-dimensional variability on multiple time scales using Multi-Scale Correlation Analysis. Peaks of the $E V(1 / 2, t)_{\max }$ curve that is presented in the upper panel mark the middle points of these intervals. The corresponding time scale $\tau_{\text {max }}$, which is presented in the middle panels, gives the width of these intervals. This is illustrated in the bottom panel, which shows the original multi-channel light curves of GB911118 by means of dashed lines for 3 arbitrarily selected peaks of $E V(1 / 2, t)_{\max }$

of GRB911118. The time history of $\tau_{\max }(t)$ is stepwise because for computational reasons the calculations were performed for the limited number of logarithmically spaced values: $\tau=0.064 \cdot 2^{n}$ (s) where $n=0, \ldots, 7$.

\subsection{The nature of the second and higher dimensions and hidden dynamics of spectral variability}

Figure (5) shows that there are sub-intervals of GB911118 (e.g. around $t=3 \mathrm{~s}$ and $t=7 \mathrm{~s}$ ) where the spectral variability is not one-dimensional at any time scale. There are two alternatives: i) emission during such time intervals is of a different, two-dimensional, nature or ii) emission with onedimensional spectral variability is persistent along the entire GRB but there is an additional component also with one-dimensional variability which emerges in various parts of the light curve and then becomes negligible.

In principle, a possible physical connection between emitters within one source should manifest itself via a correlation between their light curves. How- 
ever, in the case of relativistic expansion these light curves would likely be shifted in time and energy in the observer's frame. This could add extra dimensions to otherwise low-dimensional spectral variability.

To deal with this problem, we should allow lags between the individual light curves and look for the CM with the lowest rank $R_{C M}$ over the continuum of the lags. Such an analysis was done for the entire duration of GB911118 [8], and we found a combination of lags that makes $R_{C M} \sim 1$ for the entire burst.

\section{Conclusions}

We can see that the spectral variability in GRBs is at most two-dimensional, and it may be possible to reduce it even to one-dimension by introducing time lags between the light curves in different energy channels. This brings us to the hypothesis of a single emission source within a GRB with a single dominating variable physical parameter and some kind of "echoes" shifted in time and energy for geometrical reasons. A natural physical framework for modeling this behavior would be an optically thick expanding photosphere. However, this picture is in contradiction with models that involve more than one emission mechanism, e.g. Ryde et al [9].

\section{References}

[1] Chernenko, A., Mitrofanov, I.: $M N R A S, \mathbf{2 7 4}$, 361-368 (1995).

[2] Bagoly, Z., et. al.: $A \& \mathcal{E} A, 493$, Issue 1, 51-54 (2009).

[3] Chernenko, A.: in the Proceedings of the IAU 8th Asian-Pacific Regional Meeting, edited by S. Ikeuchi, J. Hearnshaw, and T. Hanawa, the Astronomical Society of Japan, 2002, pp. 321-322.

[4] Anscombe, F. J.: Biometrika, 35, 246-254 (1948).

[5] Marcenko, V. A., Pastur, L. A.: Math. USSR-Sb. 1507-1536 (1967).

[6] Iain, M., Johnstone: Ann. Statist. Volume 29, Number 2 (2001), 295-327.

[7] Tracy, C. A., Widom, H.: Comm. Math. Phys. $177727-754$.

[8] Chernenko, A.: in the Proceedings of XXIèmes Rencontres de Blois Windows on the Universe, June 21-26, 2009, Blois, France, (2009).

[9] Ryde, F., et al.: ApJLett, 625, L95-L98 (2005).

Anton Chernenko

E-mail: anton@cgrsmx.iki.rssi.ru

Space Research Institute

117 997, 84/32 Profsoyuznaya Str, Moscow, Russia 